\title{
A Bingham plastic and peripheral layer model of blood flow in the presence of stenosed artery
}

\author{
Rajesh Shrivastava $^{1 *}$, R.S. Chandel ${ }^{2}$, Ajay Kumar ${ }^{3}$, Keerty Shrivastava ${ }^{4}$ and Sanjeet Kumar ${ }^{5}$ \\ ${ }^{1,3}$ Government Science and Commerce College, Benazir Bhopal (M.P)-462016, India \\ ${ }^{2}$ Government Geetanjali Girls College, Bhopal (M.P)-462001, India \\ ${ }^{4}$ Government Post Graduate Bhel College, Bhopal (M.P)-462021, India \\ ${ }^{5}$ Lakshmi Narain College of Technology and Science, Bhopal (M.P)-462021, India \\ *Corresponding Author: rajeshraju0101@ rediffmail.com,ajaykumarrgpv@gmail.com*
}

Available online at: www.isroset.org

Received: 03/Jun/2018, Revised: 11/Jun/2018, Accepted: 24/Jun/2018, Online: 30/Jun/2018

\begin{abstract}
The present work deals with the blood flow through the stenosis assuming that flowing blood is represented by a two layered model. We considered a two-layered blood flow model to study the axisymmetric flow of blood by assuming the core layer as Bingham plastic (non-Newtonian fluid) and the peripheral layer (Newtonian fluid) in through the stenosis in the arteries. The analytical expressions for flow rate, resistance to flow and wall shear stress have been developed in this model. We have depicts the effect of stenosis on resistance to flow and wall shear stress. This study gives an insight into the effects of slip velocity on the volumetric flow rate, resistance to flow and wall shear stress.
\end{abstract}

Keywords: Stenosis, blood flow, core layer, peripheral layer and Bingham plastic AMS Subject Classification (2010): 76Z05, 92C35

\section{INTRODUCTION}

This study is related to blood flow through stenosed arteries having compiled wide interest in the field of Bio-Medical research. Atherosclerosis or stenosis may be defined as the formation of plaques in the lumen of the artery. Plaques lead to significant change in the flow of blood, the pressure distribution and the wall shear stress, by that means leading to cardiovascular diseases. Many investigations are performed for the cure of atherosclerosis which results in better understanding the characteristics of this type of disease.

Kapur [9] studied the mathematical models in biology and medicine. He described well about the blood flow behaviour in the stenosed artery. Chaturani et al. [5] have studied on the two phase model for blood flow through small diameter tubes. A mathematical model for the blood flow through narrow vessels with mild stenosis is developed by Jain et al. [8]. A mathematical model for the study of blood flow through a channel with permeable walls is given by Mishra et al. [14]. The effect of stenosis on the non-Newtonian flow of the blood in an artery by Shukla et al. [20]. Flow in Locally constricted tubes at low Reynolds numbers studied by Fung et al. [6]. The effect of time-dependent stenosis on flow through a tube is investigated by Young [24]. The study of the effects of couple stresses on the blood flow through the thin artery with mild stenosis has been developed by Sinha and Singh [21]. A mathematical model for different shapes of stenosis and slip velocity at the wall through mild stenosis artery by Kumar et al. [10]. The effect of stenosis on Bingham-Plastic flow of blood through an arterial tube studied by Maiti [13], which is show that the variation of resistance to flow with stenosis parameters. The effect of elastic property of the wall on flow characteristics through arterial stenosis studied by Moayeri et al. [15], they described well about the blood flow in the arterial system. Shrivastava [22] studied a two-phase model of blood flow through stenosed tubes in the presence of a peripheral layer: Applications. A nonlinear mathematical model of blood flow in a constricted artery experiencing body acceleration is described by Chakravarty et al. [3]. The effect of peripheral layer viscosity on blood flow through an artery mild stenosis is derived by Shukla et al. [19]. The published literature also reveals that in the stenotic portion, blood exhibits low shear rate. 


\section{MATHEMATICAL ANALYSIS}

In this paper, we considered a two-layered blood flow model to study the axisymmetric flow of blood by assuming the peripheral layer as Newtonian fluid and the core layer as Bingham plastic type non-Newtonian fluid in presence of stenosis in the arteries.

Consider the steady flow of blood through an axially symmetric but radially non-symmetric stenosed inelastic cylindrical arterial tube.

The geometry of constriction in the peripheral layer and core layer may be taken as Shrivastava et al. [23].

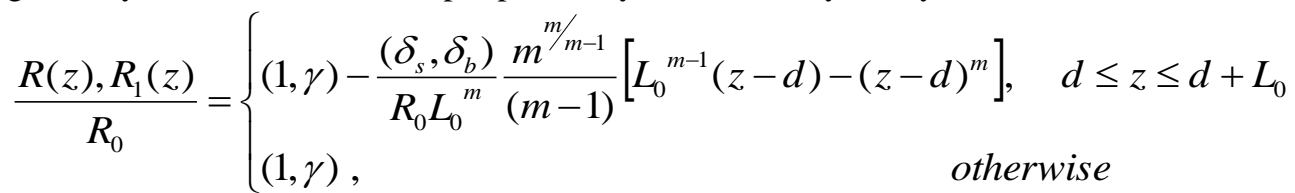

where $R(z) \approx R$ is the radius of the tube in the stenosed region, $R_{0}$ is the radius outside the stenosis, $L_{0}$ is the length of the stenosis, $d$ indicates its location and $m \geq 2$ is the shape parameter, $\gamma$ is the ratio of the central core radius to the tube radius outside the stenotic region, $\delta_{s}$ is the maximum height of the stenosis, $\delta_{b}$ is the bulging of the interface in the stenotic region located at $z=d+L_{0} / m^{1 /(m-1)}$ and $\delta / R_{0}<<1$.

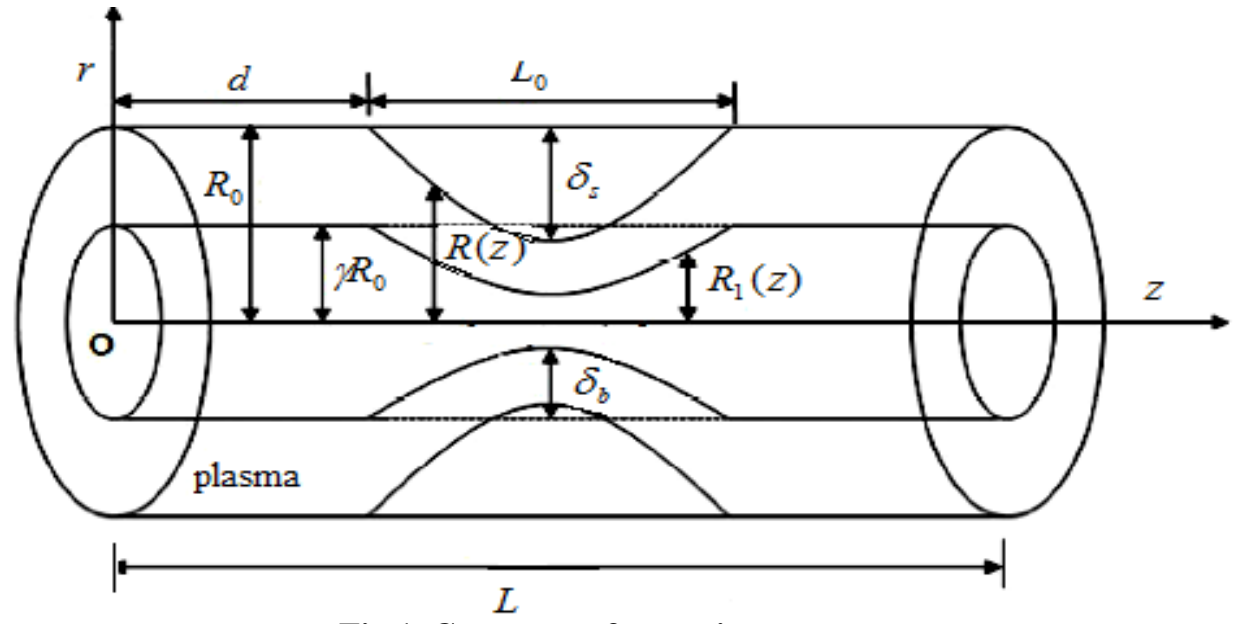

Fig.1. Geometry of stenosis

The equation of motion for laminar, incompressible steady fully developed flow is given by:

$$
\frac{d p}{d z}=\frac{1}{r} \frac{\partial(r \tau)}{\partial r}
$$

where $\frac{d p}{d z}$ is the pressure gradient and $(r, z)$ are the axial co-ordinates

The relationship between shear stress and shear rate for Bingham plastic fluid is given by:

$$
\tau=\tau^{\prime}+\mu\left(-\frac{\partial u}{\partial r}\right)
$$

where $u$ is axial velocity, $\tau$ is shear stress, $\tau^{\prime}$ is the yield stress, and $\mu$ is the viscosity of the blood.

\section{Boundary Conditions:}

The velocity boundary condition on the arterial wall is the no-slip condition, given by:

$$
u=u_{s} \quad \text { at } r=R(z)
$$

The regularity condition is given by:

$\tau$ is finite at $r=0$

Integrate equation (2) and using equation (5), we get: 
$\tau=\frac{r}{2} \frac{d p}{d z}=\frac{r}{2} P$

where $P$ is $d p / d z$.

Flow rate: The volumetric flow rate $Q_{c}$ at the core layer is given by:

$Q_{c}=\int_{0}^{R_{1}} 2 \pi r u d r=\pi \int_{0}^{R_{1}} r^{2}\left(-\frac{\partial u}{\partial r}\right) d r$

using equations (3), (4) and (6) into (7), we get:

$$
\begin{aligned}
& Q_{c}=\pi \int_{0}^{R_{1}} \frac{r^{2}}{\mu_{c}}\left\{\left(\frac{r}{2} P\right)-\tau^{\prime}\right\} d r \\
& Q_{c}=\pi \int_{0}^{R_{1}} \frac{r^{2}}{\mu_{c}}\left\{\left(\frac{r}{2} P\right)-\tau^{\prime}\right\} d r \\
& Q_{c}=\frac{\pi}{\mu_{c}}\left\{\frac{R_{1}^{4}}{8} P-\frac{R_{1}^{3} \tau^{\prime}}{3}\right\}
\end{aligned}
$$

where $\mu_{c}$ is the viscosity of the central core layer

The volumetric flow rate $Q_{p}$ at the plasma layer is given by:

$Q_{p}=\int_{R_{1}}^{R} 2 \pi r u d r=\int_{R_{1}}^{R} \frac{\pi r^{2}}{2 \mu_{p}} P d r$

using equations (3), and boundary conditions, we get:

$Q_{p}=\frac{1}{8} \frac{\pi P}{\mu_{p}}\left(R^{4}-R_{1}^{4}\right)$

where $\mu_{p}$ is the viscosity of the plasma core layer

The total flow rate is given by:

$Q=Q_{c}+Q_{p}=\frac{1}{8} \frac{\pi}{\mu_{p}}\left[P\left\{R^{4}-(1-\mu) R_{1}^{4}\right\}-\frac{8 \tau^{\prime} \mu}{3} R_{1}^{3}\right]$

where $\mu=\mu_{p} / \mu_{c}$, and the pressure gradient $P$ can be defined as:

$P=\frac{d p}{d z}=\frac{\left(\frac{8 \mu_{p} Q}{\pi}+\frac{8 \tau^{\prime} \mu R_{1}^{3}}{3}\right)}{R^{4}-(1-\mu) R_{1}^{4}}$

Integrating equation (11) using condition $p=p_{i}$ at $z=0$ and $p=p_{0}$ at $z=L$, we get:

$$
p_{i}-p_{0}=\frac{\frac{8 \mu_{p} Q L}{\pi}+\frac{8 \tau^{\prime} \mu R_{0}^{3}}{3}\left\{\left(L-L_{0}\right) \gamma^{3}+\int_{d}^{d+L_{0}}\left(\frac{R_{1}}{R_{0}}\right)^{3} d z\right\}}{R_{0}{ }^{4}\left\{\left(L-L_{0}\right)-\left(L-L_{0}\right)(1-\mu) \gamma^{4}\right\}\left[\int_{d}^{d+L_{0}}\left\{\left(\frac{R}{R_{0}}\right)^{4}-(1-\mu)\left(\frac{R_{1}}{R_{0}}\right)^{4}\right\} d z\right]}
$$

Resistance to flow: The resistance to flow is given by: 
$\lambda=\frac{p_{i}-p_{0}}{Q}$

The resistance to flow for Newtonian flow is given by:

$\lambda_{0}=\frac{8 \mu_{p} L}{\pi R_{0}{ }^{4}}$

The non-dimensional resistance to flow is given by:

$$
\bar{\lambda}=\frac{\lambda}{\lambda_{0}}=\frac{1+\frac{\tau_{0} \mu \pi R_{0}^{3}}{3 \mu_{p} Q}\left[\left(1-\frac{L_{0}}{L}\right) \gamma^{3}+\int_{d}^{d+L_{0}}\left(\frac{R_{1}}{R_{0}}\right)^{3} d z\right]}{\left(L-L_{0}\right)\left[1-(1-\mu) \gamma^{4}\left[\int_{d}^{d+L_{0}}\left\{\left(\frac{R}{R_{0}}\right)^{4}-(1-\mu)\left(\frac{R_{1}}{R_{0}}\right)^{4}\right\} d z\right]\right.}
$$

Wall shear stress: The wall shear stress is given by:

$$
\tau_{s}=\frac{\left[4 \frac{\mu_{p} Q R}{\pi}+\frac{4}{3} \tau_{o} \mu R_{1}^{3} R\right]}{R^{4}-(1-\mu) R_{1}^{4}}
$$

The wall shear stress for Newtonian fluid in the absence of stenosis is given by:

$$
\tau_{n}=\frac{4 \mu_{p} Q}{\pi R_{0}{ }^{4}}
$$

The wall shear stress in dimensionless from can be written as:

$$
\bar{\tau}=\frac{\tau_{s}}{\tau_{n}}=\frac{\pi R_{0}{ }^{4}\left[\frac{\mu_{p} Q R}{\pi}+\frac{1}{3} \tau_{o} \mu R_{1}^{3} R\right]}{\mu_{p} Q\left[R^{4}-(1-\mu) R_{1}{ }^{4}\right]}
$$

The wall shear stress at the throat of the stenosis is given by:

$$
\tau_{w s}=\frac{\left[4 \frac{\mu \mu_{c} Q}{\pi R_{0}{ }^{3}}+\frac{4}{3} \tau_{o} \mu\left(\alpha-\frac{\delta_{s}}{R_{0}}\right)^{3}\left(1-\frac{\delta_{s}}{R_{0}}\right)\right]}{\left[\left(1-\frac{\delta_{s}}{R_{0}}\right)^{4}-(1-\mu)\left(\alpha-\frac{\delta_{s}}{R_{0}}\right)^{4}\right]}
$$

Thus the non-dimensional wall shear stress at the throat of the stenosis can be written as:

$$
\bar{\tau}_{n d}=\frac{\tau_{w s}}{\tau_{n}}=\frac{\pi R_{0}^{4}\left[\frac{\mu \mu_{c} Q}{\pi R_{0}{ }^{3}}+\frac{1}{3} \tau_{o} \mu\left(\alpha-\frac{\delta_{s}}{R_{0}}\right)^{3}\left(1-\frac{\delta_{s}}{R_{0}}\right)\right]}{\mu Q\left[\left(1-\frac{\delta_{s}}{R_{0}}\right)^{4}-(1-\mu)\left(\alpha-\frac{\delta_{s}}{R_{0}}\right)^{4}\right]}
$$

\section{RESULT AND DISCUSSION}

The present model has been developed to analyse the effect the variation of flow rate, resistance to flow, wall shear stress and wall shear stress at throat with respect to stenosis size. Fig. 2 shows the variation of flow rate $Q$ for different values of 
$\gamma$, which shows that the flow rate $Q$ decreases with the increases of $\gamma$. Fig. 3 shows that the flow rate $Q$ decreases with the increase of $\mu$. Fig. 4 and 5 represent the variation of non-dimensional resistance to flow $\bar{\lambda}$ with respect to stenosis size $\delta_{s} / R_{0}$ which shows that resistance to flow $\bar{\lambda}$ increases with the increase of $\gamma, m$. Fig. 6 show that the resistance to flow $\bar{\lambda}$ increases with the increase of $\mu$. The variation of the non dimensional wall-shear stress $\bar{\tau}$ for different values of $\gamma$ and $\mu$ is plotted in fig. 7 and 8, which show that the wall-shear stress $\bar{\tau}$ increases with the increase of $\gamma$ but the reverse effect happen when $\mu$ increase. The wall shear stress at the throat of the stenosis $\bar{\tau}_{n d}$ for the variation of $\gamma$ and $\mu$ with respect to stenosis size $\delta_{s} / R_{0}$ is represented in fig. $9 \& 10$, which show that the wall shear stress at throat at stenosis $\bar{\tau}_{n d}$ is increases with the increase of $\gamma$ and $\mu$.

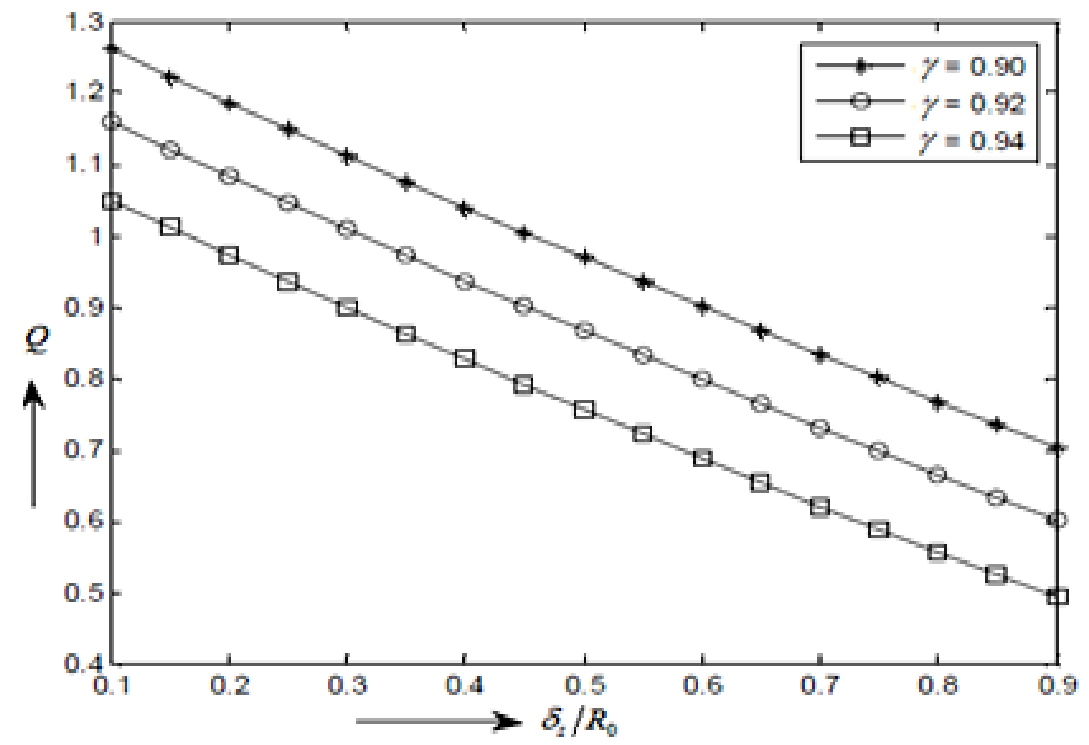

Fig. 2 variation of flow rate $Q$ with the stenosis size $\delta_{s} / R_{0}$.

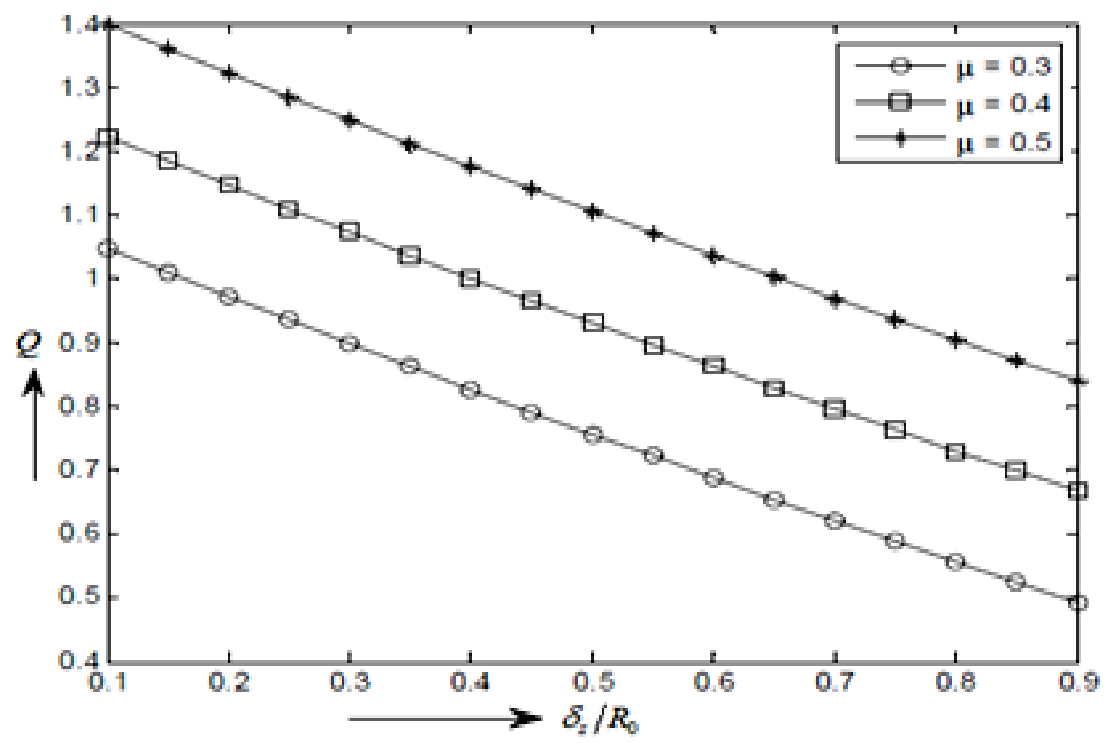

Fig. 3 variation of flow rate $Q$ with the variation of $\mu$ 


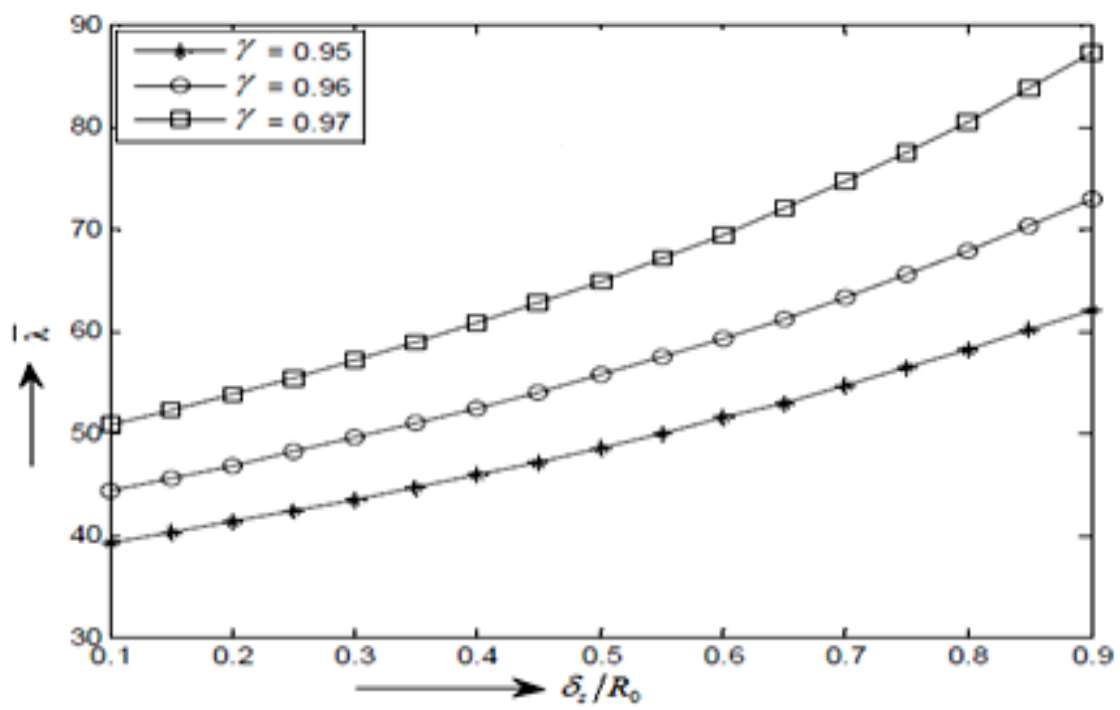

Fig. 4 variation of resistance to flow $\bar{\lambda}$ for different values of $\gamma$.

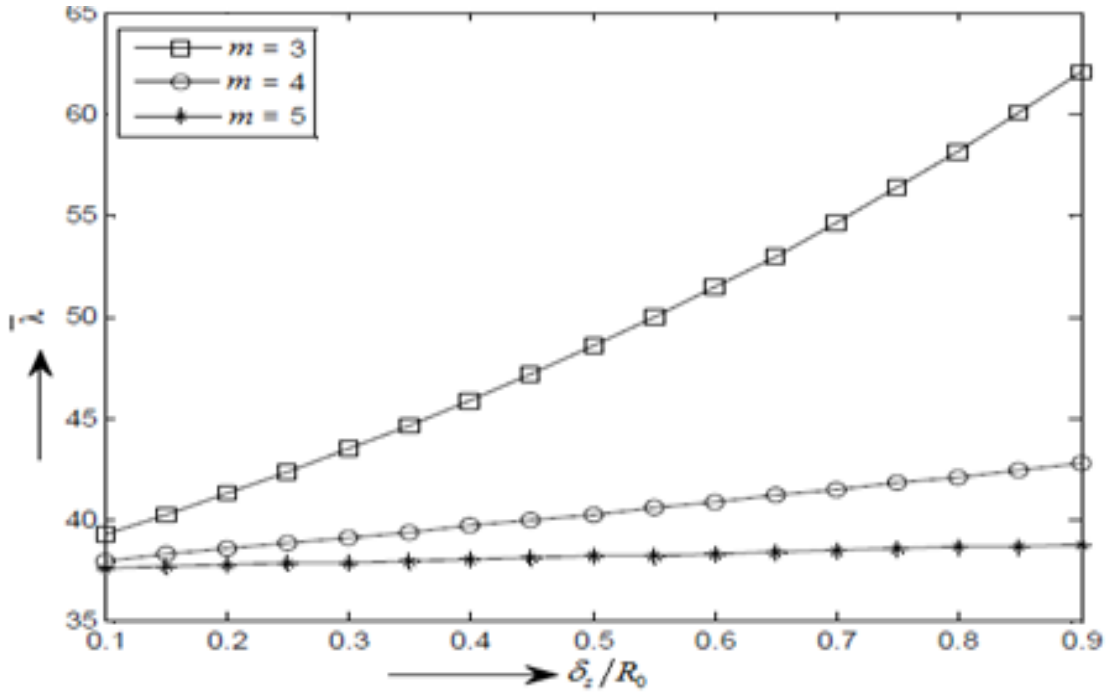

Fig. 5 variation of resistance to flow $\bar{\lambda}$ for different values of shape parameter $m$.

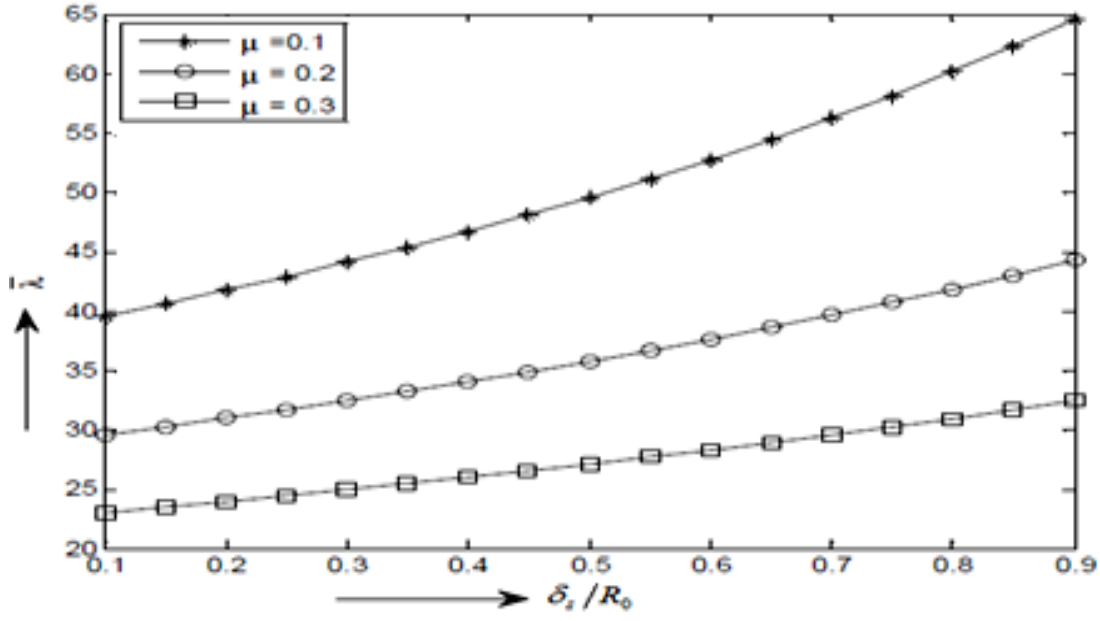

Fig. 6 variation of resistance to flow $\bar{\lambda}$ for different values of $\mu$. 


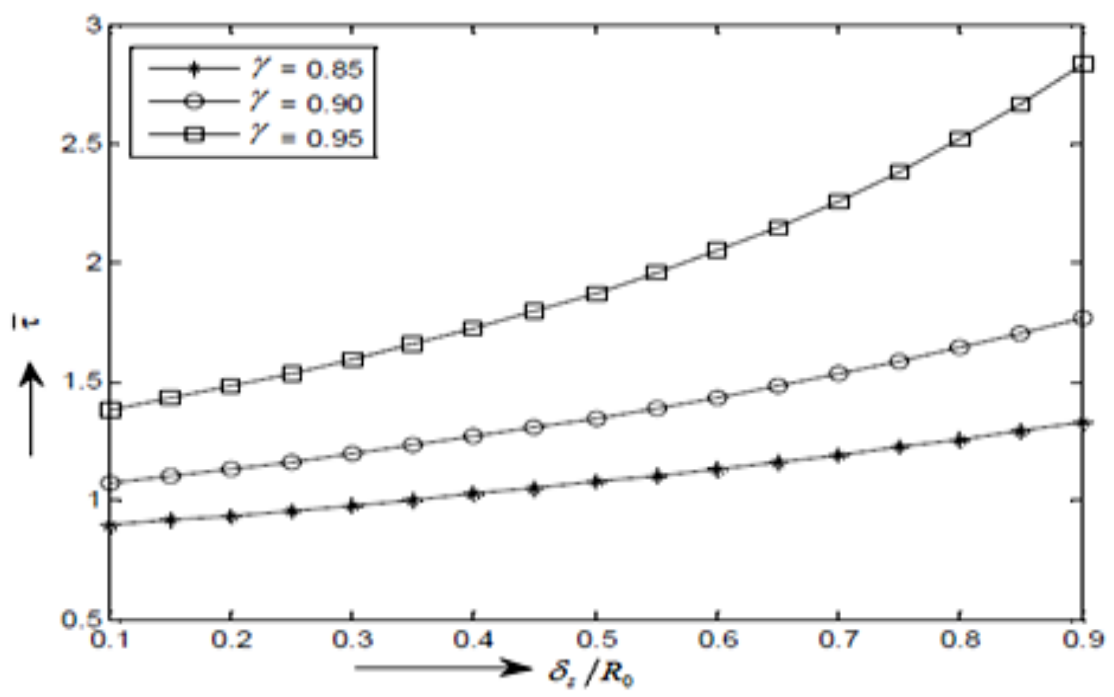

Fig. 7 The variation of wall shear stress $\bar{\tau}$ for different values of $\gamma$.

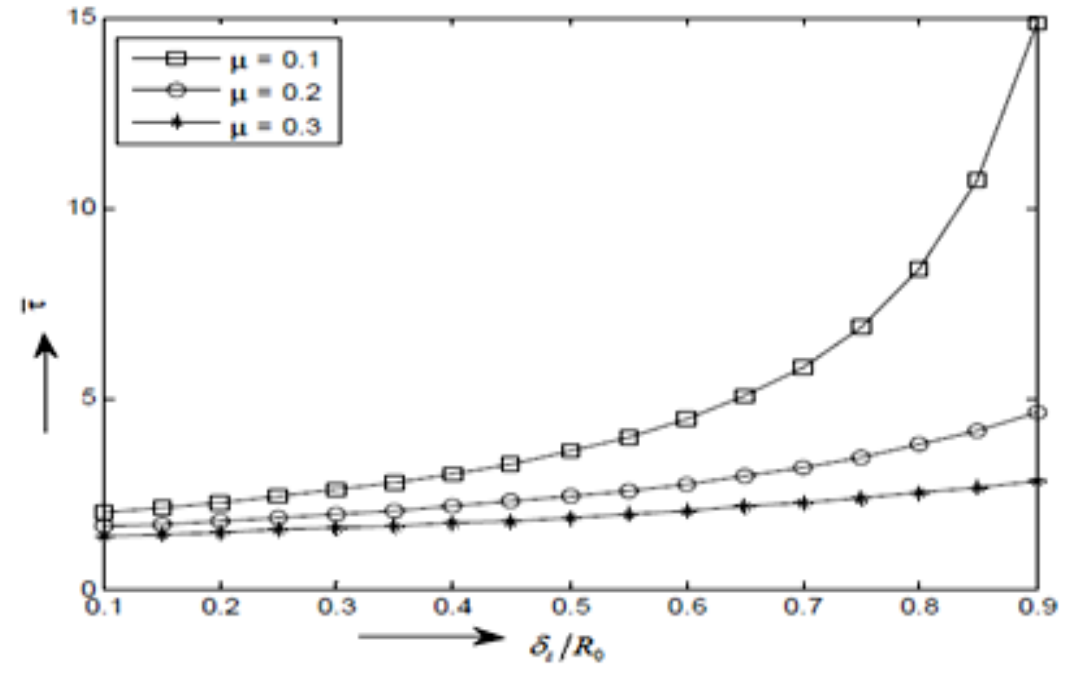

Fig. 8 The variation of wall shear stress $\bar{\tau}$ for different values of $\mu$.

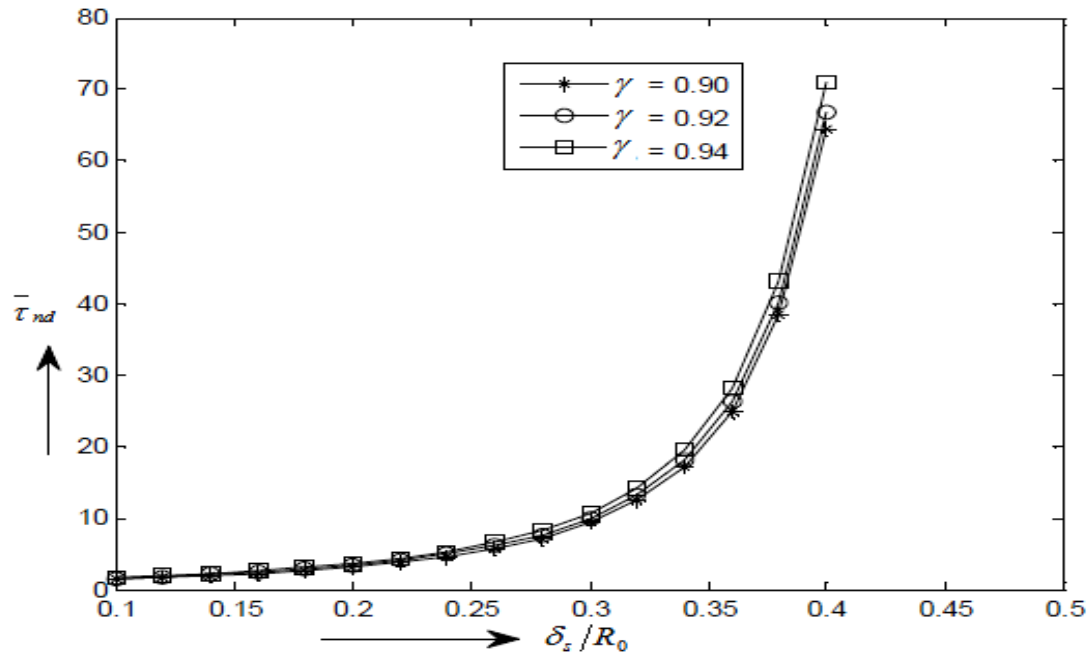

Fig. 9 The variation of wall shear stress $\bar{\tau}$ for different values of $\mu$. 


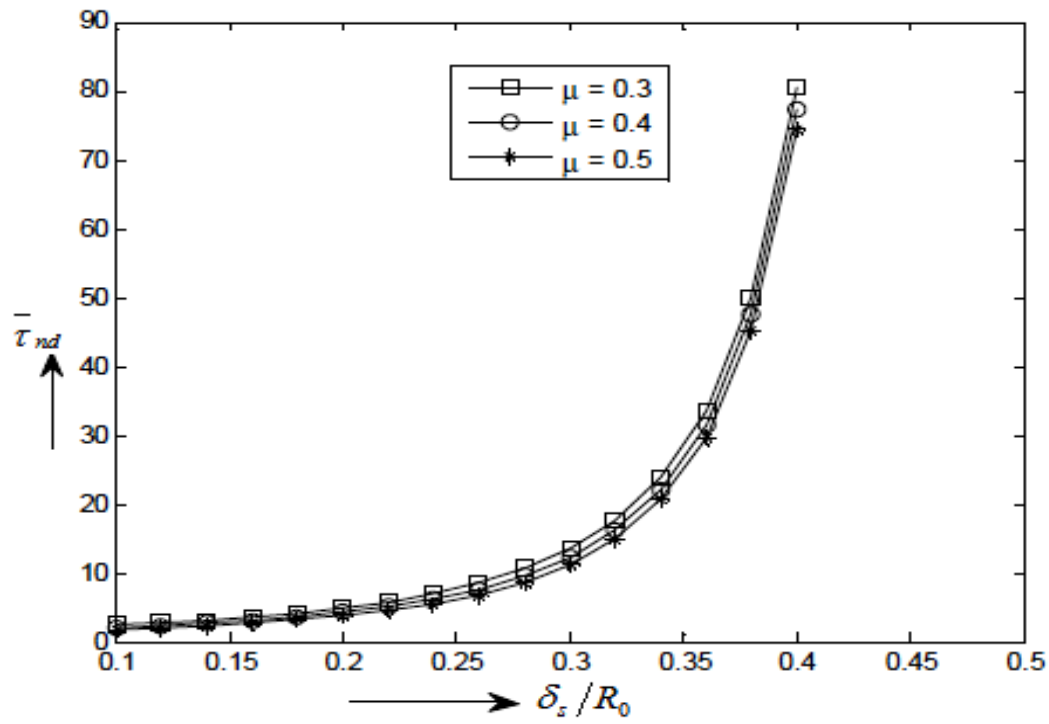

Fig. 10 The variation of non-dimensional wall shear stress at the throat of the stenosis $\bar{\tau}_{n d}$ for different values of $\mu$.

\section{CONCLUSION}

In this paper, we constructed a two-layered blood flow model to study the core layer as Bingham plastic (non-Newtonian fluid) and the peripheral layer as the Newtonian fluid through the stenosis in the arteries. We have defined the effect of stenosis on resistance to flow and wall shear stress. These results are derived from the numerical solutions using MATLAB software for distinct values which are shown by graphically (fig.2-10) and we described as follows,

i) The flow rate decreases as stenosis shape parameter with the increases of $\gamma, \mu$.

ii) The resistance to flow $\bar{\lambda}$ increases with the increase of $\gamma, m$.

iii) The wall shear stress at the throat at stenosis $\bar{\tau}_{n d}$ is increases with the increase of $\gamma$ and $\mu$.

The study of blood flow could be useful in blood flow modeling, its accurate and exact measure in accordance with physiological situations, is quite important.

\section{REFERENCES}

[1] B.S. Bhatt and N.C. Sachet, "On the analogy in slip flows", Indian Journal of Pure and Applied Mathematics., Vol. 10, pp. 303-306, 1979.

[2] G. Bulugliarello and J.W. Hyden, "Detailed characteristics of the flow of blood in vitro", Trans. Soc. Rheology, Vol. 7, pp. $209,1963$.

[3] S. Chakravarty and Kr. Sannigrahi, "A nonlinear Mathematical model of blood flow in a constricted artery experiencing body acceleration" Mathematical and Computer Modelling, Vol. 29, pp. 9-25, 1999.

[4] S. Chakravarty, A. Datta and A. K. Mandal, "Effect of stenosis on the flow behaviour of blood in an artery", IJES, Vol. 25, pp. 1003-1018 1987.

[5] P. Chaturani and Upadhyay, "Two phase model for blood flow through small diameter tubes", Biorheology, Vol. 16, pp. 109-118, 1979.

[6] Y.C. Fung and J.S. Lee, "Flow in Locally constricted tubes at low Reynolds numbers", J. Appl. Mech., Trans. ASME, Vol. 37, pp. 9, 1970,

[7] K. Haldar, "Effect of the shape of stenosis on the resistance to blood flow through an artery", Bulletian of Mathematical Biology, Vol. 47, pp. $545-550,1985$

[8] M. Jain, G. C. Sharma and S. Kumar, "A mathematical model for the blood flow through narrow vessels with mild stenosis", IJE, Vol. 1, pp. 99106, 2009.

[9] Kapur, J.N, "Mathematical Models in Biology and Medicine", Affiliated East-Wes press, New Delhi. 1985.

[10] H. Kumar, R.S. Chandel, S. Kumar and S. Kumar, "A mathematical model for different shapes of stenosis and slip velocity at the wall through mild stenosis artery", Advances in Applied Mathematical Biosciences, Vol.5, No. 1, pp. 9-18, 2014.

[11] D. A. MacDonald, "Blood flow in Arteries", Edward Arnold, Londan, 1974.

[12] D. A. MacDonald, "On steady flow through modeled vascular stenosis" J. Biomechanics, Vol. 12, pp. 13-30, 1979.

[13] A. K. Maiti, "Effect of stenosis on Bingham-Plastic flow of blood through an arterial tube" Institute Journal of Mathematics trends and Technology, Vol. 13, No 1, pp. 50-57, 2014.

[14] J.C. Mishra and S.K Ghosh, "A mathematical model for the study of blood flow through a channel with permeable walls", Acta. Mechanica, Vol. 12, pp. 137-153, 1997. 
[15] M.S. Moayeri and Zendehbudi, "The effect of elastic property of the wall on flow characteristics through an arterial stenosis", J. of Biomechanics, Vol. 36, pp. 525-535, 2003.

[16] R.K. Naeem, S. Younus and Dania, "Inverse solutions for unsteady in-compressible couple fluid flow", IJAMM, Vol. 6, pp. 1-17, 2010.

[17] G. Radhakrishnamacharya and P. Shrinivasa Rao, "Flow of a magnetic fluid through a non-uniform wavy tube", PNASI, Vol. 76, pp. 241-245, 2007.

[18] B.R.P Rao, "Effect of stenosis on non-Newtonian flow of the blood in an artery", Bull. Math., Vol. 42, pp. 283-294, 1980.

[19] J.B., Shukla, R.S. Parihar and B.R.P. Rao, "Effect of peripheral layer viscosity on blood flow through an artery mild stenosis", Bull Math. Bio, Vol. 17, pp. 403-410, 1980.

[20] J.B. Shukla, R.S. Parihar and B.R.P. Rao, "Effects of stenosis on non-Newtonian flow of the blood in an artery", Bulletin of Math. Biology, Vol. 42, pp. 283-294, 1980 (b).

[21] P Sinha and C. Singh, "Effect of couple stresses on the blood flow through an artery with mild stenosis", Biorheology, vol. 15, pp. 3-4, 1984.

[22] V.P. Srivastava, "Two-phase model of blood flow through stenosed tubes in the presence of a peripheral layer: Applications", Journal of Biomechanics, Vol. 29, No. 10, pp. 1377-1382, 1996.

[23] V.K. Stokes, "Couple stresses in fluids, Fluid, Vol. 9, pp. 1710, (1966).

[24] D.F. Younge, "Effects of time-dependent stenosis on flow through a tube”, J. Engrg. Ind. Trans. ASME, Vol. 90, pp. 248-254, 1968. 